\title{
FrUITeR: A Framework for Evaluating UI Test Reuse
}

\author{
Yixue Zhao \\ yixue.zhao@usc.edu \\ University of Southern California, USA \\ Marcelo Schmitt Laser \\ marcelo.laser@gmail.com \\ University of Southern California, USA
}

\author{
Justin Chen \\ justin.chen@columbia.edu \\ Columbia University, USA \\ Jie Zhang \\ jie.zhang@ucl.ac.uk \\ University College London, UK
}

\author{
Adriana Sejfia \\ sejfia@usc.edu \\ University of Southern California, USA \\ Federica Sarro \\ f.sarro@ucl.ac.uk \\ University College London, UK
}

\author{
Mark Harman \\ mark.harman@ucl.ac.uk \\ University College London, UK
}

\author{
Nenad Medvidovic \\ neno@usc.edu \\ University of Southern California, USA
}

\begin{abstract}
UI testing is tedious and time-consuming due to the manual effort required. Recent research has explored opportunities for reusing existing UI tests from an app to automatically generate new tests for other apps. However, the evaluation of such techniques currently remains manual, unscalable, and unreproducible, which can waste effort and impede progress in this emerging area. We introduce FrUITeR, a framework that automatically evaluates UI test reuse in a reproducible way. We apply FrUITeR to existing test-reuse techniques on a uniform benchmark we established, resulting in 11,917 test reuse cases from 20 apps. We report several key findings aimed at improving UI test reuse that are missed by existing work.
\end{abstract}

\section{CCS CONCEPTS}

- Software and its engineering;

\section{KEYWORDS}

Software Testing, Test Reuse, Mobile Application, Open Science

\section{ACM Reference Format:}

Yixue Zhao, Justin Chen, Adriana Sejfia, Marcelo Schmitt Laser, Jie Zhang, Federica Sarro, Mark Harman, and Nenad Medvidovic. 2020. FrUITeR: A Framework for Evaluating UI Test Reuse. In Proceedings of the 28th ACM foint European Software Engineering Conference and Symposium on the Foundations of Software Engineering (ESEC/FSE '20), November 8-13, 2020, Virtual Event, USA. ACM, New York, NY, USA, 12 pages. https://doi.org/10.1145/ 3368089.3409708

\section{INTRODUCTION}

Writing UI tests is tedious and time-consuming [23, 26], increasingly driving the focus toward automated UI testing [22]. However, existing work tends to target tests that yield high code coverage, rather than usage-based tests that explore an app's functionality,

Permission to make digital or hard copies of all or part of this work for personal or classroom use is granted without fee provided that copies are not made or distributed for profit or commercial advantage and that copies bear this notice and the full citation on the first page. Copyrights for components of this work owned by others than ACM must be honored. Abstracting with credit is permitted. To copy otherwise, or republish, to post on servers or to redistribute to lists, requires prior specific permission and/or a fee. Request permissions from permissions@acm.org.

ESEC/FSE '20, November 8-13, 2020, Virtual Event, USA e.g., sign-in, purchase, search, etc. Developers heavily rely on usagebased tests [26], but currently have to write them manually [22, 26].

To reduce the manual effort of writing usage-based tests, recent research has explored reusing existing tests in a source app to generate new tests automatically for a target $a p p$ [20, 21, 23, 25, 28]. The guiding insight is that different apps expose common functionalities via semantically similar GUI elements. This suggests that it is possible to reuse existing UI tests across apps-in effect generating the tests automatically-by mapping similar GUI elements.

Four recent techniques have targeted usage-based test reuse across Android apps [20, 21, 23, 25]. ${ }^{1}$ While these techniques have shown promise, we have identified five important limitations that hinder their comparability, reproducibility, and reusability. In turn, this can lead to duplication and wasted effort in this emerging area.

(1) The metrics applied to date evaluate whether GUI events from a source app are correctly transferred to a target app, but do not consider whether the transferred tests are actually useful. It is possible that events are transferred correctly, but the generated test is "wrong". This can be, e.g., because a generated test is missing events and thus not executable. Moreover, the metrics used in existing work are not standardized even when evaluating same aspects of different techniques, making it difficult to compare the techniques.

(2) Each existing technique's evaluation process requires significant manual effort: every transferred event in each test must be inspected to determine whether the transfer is performed correctly. This imposes a practical limit on the number of tests that can be evaluated. For instance, the authors of ATM [21] had to restrict their comparison with GTM [20] to a randomly selected $50 \%$ of the possible source-target app combinations due to the task's scale.

(3) There are no standardized guidelines for conducting the manual inspections, making the evaluation results biased and hard to reproduce. For instance, ATM's authors acknowledge the possibility of mistakes in the manual process [21]. Such mistakes are currently hard to locate, verify, or eliminate by other researchers.

(4) Existing techniques are designed as one-off solutions and evaluated as a whole. This makes it difficult to isolate and compare their relevant components. For instance, GTM [20], ATM [21], and CraftDroid [25] all contain functionality to compute a "similarity score"

\footnotetext{
${ }^{1}$ Rau el al. recently proposed a test-reuse technique for web applications [28]. In this paper, we focus on Android apps due to the availability of a larger number of existing techniques to evaluate, although in principle our work is not limited to Android.
} 
between two GUI elements, but it is unclear which of those specific components performs the best against the same baseline. This would impede subsequent research that could potentially benefit from identifying underlying components that should be reused and/or improved.

5 Existing techniques make different assumptions that hinder their comparison. For instance, GTM [20] and ATM [21] require access to apps' code, and cannot be directly compared with techniques evaluated on close-sourced apps.

To address limitations $1-3$, as well as limitation 4 in part, we have developed

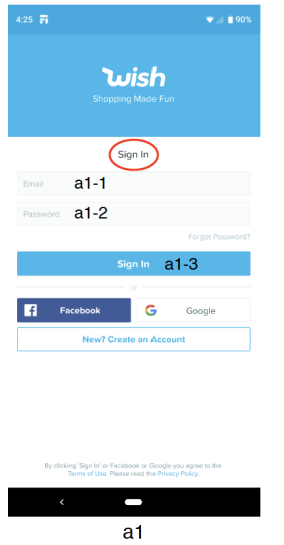
FrUITeR, a Framework for evaluating UI Test Reuse. FrUITeR consists of three key elements: a set of new evaluation metrics that consolidate the metrics used by existing techniques and expand them to measure important aspects that are currently missed; $t$ wo baseline UI test-reuse techniques that establish the lower- and upperbounds for the evaluation metrics; and an automated workflow that modularizes UI test-reuse functionality and significantly reduces the manual effort. With FrUITeR, one can automatically evaluate testreuse techniques on apps/tests of interest against the same baseline, thus opening the possibility of in-depth studies at a large-scale.

To fully address limitation 4 , as well as limitation 5 , we have extracted the core components from existing techniques and established a benchmark for evaluating and comparing them. Our benchmark currently contains 20 subject apps with 239 test cases, involving 1,082 GUI events. This benchmark is used by FrUITeR to evaluate side-by-side the extracted components and the two baseline components we developed, yielding 11,917 test-reuse instances.

The results obtained by FrUITeR revealed several important findings. For example, we have been able to pinpoint specific tradeoffs between ML-based (e.g., AppFlow) and similarity-based (e.g., ATM) techniques. We have also identified scenarios that may seem counter-intuitive, such as the fact that manually writing tests requires less effort than attempting automated transfer in certain cases. Finally, performing evaluations on a much larger data corpus allowed us to refute some conclusions reached in prior work.

This paper makes the following contributions. (1) We develop FrUITeR to automatically evaluate UI test reuse with an expanded set of metrics as compared to existing work, and two baseline techniques that help to provide the lower- and upper-bounds of UI test reuse in a given scenario. (2) We identify and extract the core components from existing test-reuse techniques, enabling their fair comparison. 3 We establish a reusable benchmark with standardized ground truths that facilitates the reproducibility of UI test-reuse techniques' evaluation and comparison. (4) We use FrUITeR to conduct a side-by-side evaluation of the state-of-the-art test-reuse techniques, uncovering several needed improvements in this area. 5 We make FrUITeR's implementation and all data artifacts publicly available [12], directly fostering future research.

Section 2 introduces a representative example, terminology, and related work. Section 3 describes FrUITeR's requirements and Section 4 its design, followed by FrUITeR's instantiation in Section 5. Section 6 discusses our key findings. Section 7 concludes the paper.

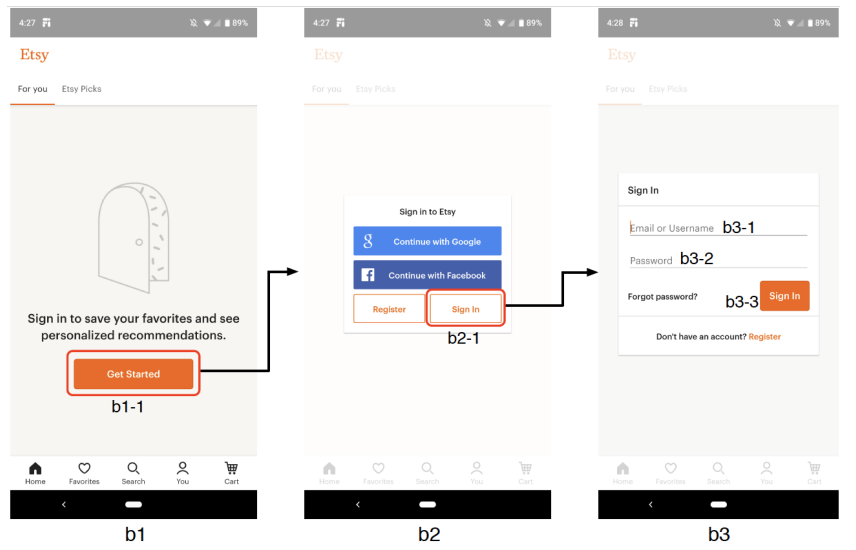

Figure 1: sign-in tests for Wish (a1) and Etsy (b1-b3).

\section{BACKGROUND AND RELATED WORK}

In this section, we introduce a motivating example and relevant terminology, followed by an overview of the strategies pursued by existing work and how they have been evaluated to date.

\subsection{Motivating Example and Terminology}

Figure 1 shows the screenshots of the sign-in process of two popular shopping apps: Wish (left) and Etsy (right). Each screen is labeled with an identifier, e.g., a1 is the first screen of Wish. In each screen, there may be one or more actionable GUI elements with which end-users can interact based on the associated actions. For instance, the "Sign In" button in screen a1 (a1-3) is associated with a click action. Actionable elements and their associated actions embody GUI events (defined below). By contrast, the label "Sign In" that is circled in screen a 1 is a non-actionable GUI element.

As an illustration, assume that Wish's sign-in test exists and our goal is to automatically transfer it to Etsy. The relevant actionable GUI elements in this sign-in example are labeled and will be used to describe the following key terms used throughout the paper.

GUI Event, or event in short, is a triple comprising (1) an actionable GUI element, (2) an associated action, and (3) an optional input value (e.g., user input for a text box). We reuse this definition from existing work [20,21, 25]. For simplicity, we use the label of a GUI element (e.g., a1-1) to refer to the GUI event triple.

Canonical Event is an abstracted event that captures a category of commonly occurring events. An example canonical event may be AppSignIn, and it would correspond to the a1-3 and b3-3 from Figure 1, as well as similar events from other apps, such as Log In.

Usage-Based Test exercises a given functionality in an app, such as sign-in. A usage-based test ${ }^{2}$ consists of a sequence of GUI events. For instance, Figure 1 highlights the sign-in test in Wish (left) as the event sequence $\{$ a 1-1, a 1-2, a1-3 $\}$.

Source App is the app with known tests that can be transferred to other apps with similar usage. For instance, Wish is a source app with a sign-in test that can potentially be transferred to other apps with sign-in functionality. Target App is the app to which one aims to transfer existing tests. A target app can reuse the tests from multiple source apps; at the same time, it can serve as a source

${ }^{2}$ If not mentioned otherwise, test or test case refers to usage-based test in this paper. 
app to other target apps if it contains known tests. Both source apps and target apps are used extensively in existing work [20,21, 25].

Source Test is an existing test for a given source app that should be transferred to a target app to generate a Transferred Test. Ground-Truth Test is an existing test for a target app that is used to evaluate whether the transferred test is correct. (i.e., whether the two tests match). Source Event, Transferred Event, and Ground-Truth Event refer to the GUI events that belong to the source test, transferred test, and ground-truth test, respectively.

Ancillary Event is a special type of transferred event that is not mapped from a source event, but is added in order to reach certain states in the target app. For example, b1-1 and b2-1 from Figure 1 may need to be added as ancillary events in order to reach Etsy's sign-in screen b3; such events do not exist in the source test.

Null Event is an event that should have been mapped from a source event, but was not identified as such by a given test-reuse technique. Thus, the null event does not exist in the transferred test, but it has a corresponding source event from which it maps. This could be because of (1) a test-reuse technique's inaccuracy or (2) the difference in app behaviors. An example of the latter would be the inability to map Etsy's events b1-1 and b2-1 to Wish in Figure 1.

\subsection{Strategies Explored to Date}

Four recent techniques [20,21, 23, 25] have targeted UI test reuse in Android. The shared core concern of these techniques is to correctly map the GUI events from a source app to a target app. In the example from Figure 1, the source test sign-in in Wish comprises the event sequence $\{a 1-1$, a1-2, a1-3\}. By mapping GUI events in this test from Wish to Etsy as $\{$ a 1-1 $\rightarrow$ b3-1, a1-2 $\rightarrow$ b3-2, a1-3 $\rightarrow$ b3-3\}, a new sign-in test for the target app, Etsy, is generated as $\{b 3-1, b 3-2, b 3-3\}$.

The existing techniques can be classified into two main categories, based on how they map GUI events across different apps: AppFlow [23] is an ML-based, while CraftDroid [25], GTM [20], and ATM [21] are similarity-based techniques. We have abstracted the two categories and their underlying workflows through studying the similarities and differences across the existing techniques.

ML-based techniques learn a classifier from a training dataset of different apps' GUI events based on certain features, such as text, element sizes, and image recognition results of graphical icons. The classifier is used to recognize app-specific GUI events and map them to canonical GUI events used in a test library, so that app-specific tests can be generated by reusing the tests defined in the test library.

Similarity-based techniques define their own algorithms to compute a similarity score between pairs of GUI events in a source app and a target app based on the information extracted from the two apps, such as text, element attributes, and Android Activity/Fragment names. The similarity score is used to determine whether there is a match between each GUI event in the source app and the one in the target app based on a customizable similarity threshold. For example, a 1-1 in Wish (left) from Figure 1 is likely to have a higher similarity score with b3-1 than with other GUI events in Etsy (right). In that case, a1-1 in Wish will be mapped to b3-1 in Etsy. Another important component in similarity-based techniques is the exploring strategy, which determines the order of computing the similarity score between the GUI events in the source and target apps. The target app's events that are explored earlier usually have a higher chance of being mapped.

\subsection{Existing Evaluation Metrics}

To evaluate their test-reuse strategies, existing techniques have focused on the accuracy of the GUI event mapping. This section overviews the metrics they applied, which guided us in defining the expanded set of FrUITeR's metrics (see Section 4.1.1). Note that the exact definitions of existing metrics were not provided in the prior publications $[20,21,23,25]$; we had to separately contact the authors of each technique to obtain the details introduced below.

AppFlow [23] is an ML-based technique that maps app-specific events to canonical events using a classifier as discussed earlier. AppFlow's classifier is evaluated with the standard accuracy metric [27], indicating the percentage of the correctly-classified GUI events among all the GUI events being classified. Correctly-classified GUI events include two cases: (1) the app-specific events that are mapped to the correct canonical events (true positive); and (2) the app-specific events that are not mapped to any canonical events and such canonical events do not exist (true negative).

CraftDroid [25] is a similarity-based technique. After the transfer of events from a source app to events in a target app, CraftDroid's authors manually identify three cases: (1) true positive (TP) occurs when the transferred event is the same as the one obtained during a manual transfer; (2) false positive (FP) occurs when the transferred event is different; and (3) false negative (FN) occurs when CraftDroid fails to find a matching event, while the manual transfer succeeds. Precision and recall are then calculated based on the three cases. It is important to note that CraftDroid's FP includes both the incorrectly transferred events and the newly added ancillary events (if any), which is different from the FP case defined in other techniques. We further illustrate this in Section 4.1.1.

ATM [21] and GTM [20] are also similarity-based techniques, and ATM is an enhancement of GTM by the same authors. Similar to CraftDroid, the authors manually inspect the transferred results and identify four cases: (1) correctly matched means the source event is mapped to the correct event in the target app (TP); (2) incorrectly matched means the source event is mapped to the wrong event in the target app (FP); (3) unmatched ('exist) means the source event is not mapped to any events and no such events exist in the target app (TN); (4) unmatched (exist) means the source event is not mapped to any events although the matching event exists in the target app (FN). ATM and GTM do not calculate the precision or recall, but present the raw percentages of each of the four cases.

\section{$3 \quad$ FrUITeR's PRINCIPLE REQUIREMENTS}

This section elaborates on the key limitations of current test-reuse techniques and their evaluation processes, initially outlined in Section 1 . These limitations serve as the foundation of five principle requirements we focused on in FrUITeR's design (Section 4) and instantiation (Section 5).

Prior to developing FrUITeR, we investigated the existing techniques and their evaluations [20, 21, 23, 25] in depth. Beyond consulting the available publications, we also studied the techniques' implementations and produced artifacts [1, 3, 5, 8], and engaged their authors in, at times, extensive discussions to obtain missing details and resolve ambiguities. In the end, we identified five limitations that are likely to hinder future advances in this emerging area. We base FrUITeR's principal requirements on these limitations. 
Req 1 - Metrics used by FrUITeR to evaluate test-reuse techniques shall be standardized and reflect practical utility. Existing techniques are evaluated with different, and differently applied, metrics (recall Section 2.3), which harms their side-by-side comparison. More importantly, all techniques to date have focused on whether GUI events from a source app are correctly transferred to a target app, without considering whether the transferred tests are actually meaningful and applicable in the context of the target app. It is thus possible that all GUI events are mapped correctly, but the transferred test cannot be applied, e.g., due to missing ancillary events (recall Section 2.1). None of the existing techniques are able to identify such scenarios; FrUITeR must be able to do so.

$\operatorname{Req}_{2}-$ FrUITeR's workflow shall reduce the required manual effort and thus scale to larger numbers of apps and tests than possible with current test-reuse techniques. - Existing techniques' evaluation processes require significant manual effort to inspect every transferred event in each test. For example, ATM [21] was evaluated on 4 app categories, where each category, in turn, consisted of 4 apps. On average, each app had 10 tests to be transferred and each test had 5 events. Within each app category, ATM transferred the tests of each app to the remaining 3 apps, resulting in 48 source-target app pairs in total. For each app pair, ATM's authors had to manually inspect an average of 50 transferred events (10 tests $\times 5$ events), i.e., 2,400 events in total. This is why they were forced to restrict their comparison with GTM [20] to a randomly selected half of possible source-target app pairs. FrUITeR must address this shortcoming by providing a more scalable evaluation workflow that requires markedly less manual effort.

$\mathrm{Req}_{3}$ - Evaluation results produced by FrUITeR shall be reproducible. - As discussed in Section 2.3, the current techniques' evaluation results depend on identifying the case to which each transferred event belongs (e.g., correctly matched, false positive, etc.). Such "ground-truth mappings" are determined manually. However, there are no standard guidelines for conducting inspections, making the results potentially biased and unreproducible. In Figure 1's example, it is debatable whether $\{a 1-1 \rightarrow b 3-1\}$ is correct because a 1-1 only takes the user's email, while b3-1 takes both the email and username. ATM's authors also acknowledge the possibility of mistakes in the manual process [21]. More importantly, any such mistakes are hard to locate or verify by other researchers, since the results of manual inspection and the ground-truth mappings on which they are based, are recorded in ad-hoc ways. Thus, to facilitate future research in this area, the evaluation results produced by FrUITeR must be reproducible, with a ground-truth representation that can be independently verified, reused, and modified.

$\mathrm{Req}_{4}-$ Test-reuse capabilities incorporated and evaluated by FrUITeR shall be modularized. - Despite providing similar functionality, existing test-reuse techniques are designed as one-off solutions and evaluated as a whole. This makes it difficult to reuse or compare their relevant components. In turn, it invites duplication of effort and introduces the risk of missed opportunities for advances by other researchers, and even by the techniques' own developers. To address this problem, FrUITeR must modularize each test-reuse artifact it evaluates, allow its independent (re)use, and associate the obtained evaluation results with the appropriate artifacts.

Req $_{5}$ - Benchmarks provided and applied by FrUITeR shall be reusable. - Existing test-reuse techniques have been evaluated
Table 1: Fidelity metrics as used in AppFlow [23], CraftDroid [25], ATM [21], GTM [20], and FrUITeR.

\begin{tabular}{|c|c|c|c|c|c|c|c|}
\hline & $\begin{array}{c}\text { True Pos. } \\
\text { (TP) }\end{array}$ & $\begin{array}{c}\text { False Pos. } \\
\text { (FP) }\end{array}$ & $\begin{array}{c}\text { True Neg. } \\
\text { (TN) }\end{array}$ & $\begin{array}{c}\text { False Neg. } \\
\text { (FN) }\end{array}$ & Accuracy & Precision & Recall \\
\hline \hline AppFlow & anon & anon & anon & anon & Accuracy & $d n c$ & $d n c$ \\
\hline CraftDroid & TP & FP1 & none & FN & $n o n e$ & Precision & Recall \\
\hline $\begin{array}{c}\text { ATM/ } \\
\text { GTM }\end{array}$ & $\begin{array}{c}\text { Correctly } \\
\text { Matched }\end{array}$ & $\begin{array}{c}\text { Incorrectly } \\
\text { Matched }\end{array}$ & $\begin{array}{c}\text { Unmatched } \\
\text { (!exist) }\end{array}$ & $\begin{array}{c}\text { Unmatched } \\
\text { (exist) }\end{array}$ & $d n c$ & $d n c$ & $d n c$ \\
\hline FrUITeR & Correct & Incorrect & NonExist & Missed & Accuracy & Precision & Recall \\
\hline
\end{tabular}

using different benchmark apps and tests, additionally hampering their comparison. In fact, only three subject apps were shared by two (AppFlow [23] and CraftDroid [25]) out of the four existing techniques in their evaluations. The underlying reason is the different assumptions made by the techniques. For instance, GTM and ATM rely on the Espresso testing framework [6] that requires the apps' source code. As another example, AppFlow's tests are written in a special-purpose language based on Gherkin [13] and cannot be reused by techniques that capture tests in other languages (e.g., Java, used by ATM and GTM). Thus, FrUITeR must establish a set of uniform benchmarks with reusable apps and tests that can serve as the foundation for evaluating and comparing solutions in this area.

\section{FrUITeR's DESIGN}

This section presents FrUITeR's design, with a focus on two features that address requirements $\operatorname{Req}_{1}, \mathrm{Req}_{2}, \mathrm{Req}_{3}$, and partially Req new evaluation metrics and an automated, modular workflow. We also introduce two novel test-reuse techniques to serve as baselines for bounding the existing techniques' evaluation results.

\subsection{FrUITeR's Metrics}

To address Req 1 , FrUITeR incorporates a pair of evaluation metrics: (1) fidelity focuses on how correctly the GUI events are mapped from a source app to a target app; (2) utility measures how useful the transferred tests are in practice.

4.1.1 Fidelity Metrics. As explained in Section 2.3, fidelity of the mapping has been the main focus of existing techniques, but the previous metrics have been used inconsistently. ${ }^{3}$ To form a fair playground for comparing test-reuse techniques, we investigated existing metrics in-depth. We did so by consulting available documentation and discussing the metrics with the authors of all four techniques. We standardized this information into a comprehensive set of fidelity metrics in FrUITeR, as shown in Table 1.

Table 1 presents the fidelity metrics used across the different test-reuse techniques, and their relationship to the standard metrics as defined in literature [27]. Each row shows a mapping from the names for the metrics used by each technique to the typical fidelity metrics' names indicated in the header. "anon" cells represent metrics that are not reported by a technique, but are used internally to calculate other metrics that are reported. " $d n c$ " cells represent metrics that are not calculated by a given technique, but can be determined based on other metrics used. Finally, "none" cells represent cases where a metric is not used by a technique and cannot be calculated from the available information. FrUITeR covers all seven metrics, changing several metrics' names to better reflect their application to test reuse, as will be further discussed below.

\footnotetext{
${ }^{3}$ Existing publications in this area have referred to some of these as "accuracy" metrics. We use "fidelity" to avoid confusion with a specific metric named "accuracy" defined previously in literature [27] and used by one of the techniques we studied [23].
} 
Recall from Section 2.3 that CraftDroid's FP category covers two cases: FP1 corresponds to "Incorrectly Matched" events in ATM/GTM and "Incorrect" in FrUITeR; FP2 corresponds to the ancillary events that are not considered by other techniques. FrUITeR also excludes the ancillary events from its Incorrect category because they can be benign or even needed (e.g., b1-1 and b2-1 from Figure 1), and do not reflect the fidelity of the GUI event mapping. For instance, if ancillary events were considered to be False Positives, a large number of them would result in a low Precision for the GUI event mapping. However, this would not be a meaningful measure since the ancillary events are not mapped from the source app. Such events are thus not relevant to the mapping's fidelity, but should be considered by the utility metrics, introduced next.

4.1.2 Utility Metrics. FrUITeR introduces two utility metrics to indicate how useful a transferred test is. This aspect is not considered in prior work, but is needed because a high-fidelity event mapping does not guarantee a successfully transferred test, or vice versa. For instance, a target app's ground-truth test may contain ancillary events not covered by source events, making it impossible to generate a "perfect" test by event mapping alone. On the flip side, a low-fidelity mapping may accidentally generate a "perfect" test. Thus, it is important to measure the utility with respect to the ground-truth test independently of event mapping's fidelity.

To this end, we first define an effort metric, to measure how close the transferred test is to the ground-truth test, by calculating the two tests' Levenshtein distance [24]. Levenshtein distance is widely used in NLP to measure the steps needed to transform one string into another. In our case, each step is defined as the insertion, deletion, or substitution of an event in the transferred test.

Secondly, we define a reduction metric, to assess the manual effort saved by the generation of the transferred test, compared to writing the ground-truth test from scratch:

Reduction $=(\#$ gtEvents - effort $) \div \#$ gtEvents

The value of reduction may be negative, if transforming the transferred test into the corresponding ground-truth test takes more steps than constructing such ground-truth test from scratch.

Note that each usage-based test targets a scenario with a specific flow of interest; multiple flows would result in multiple tests (e.g., sign-in from "homepage" vs. from "settings"). For each particular flow, it is possible for the ground-truth test to contain different "acceptable" ancillary events based on one's interest, which would result in multiple "acceptable" ground-truth tests. In FrUITeR's current benchmark (see Section 5.2), we manually constructed one ground-truth test for each flow with the minimal amount of ancillary events to match prior work. However, FrUITeR's ground-truth tests can be modified or extended to obtain their corresponding utility results. For instance, researchers can specify multiple "acceptable" ground-truth tests for a given flow, and measure the transferred test's utility with respect to each ground-truth test.

We acknowledge that the utility aspect (i.e., how useful a transferred test is) can be subjective depending on one's goal. Alternative utility metrics (e.g., bug-identification power, executability, code coverage) can be added to FrUITeR's customizable workflow (see Section 4.2). FrUITeR's current utility metrics specifically center around effort because they are applied to tests transferred by techniques whose end-goal is to reduce the effort of writing tests manually.

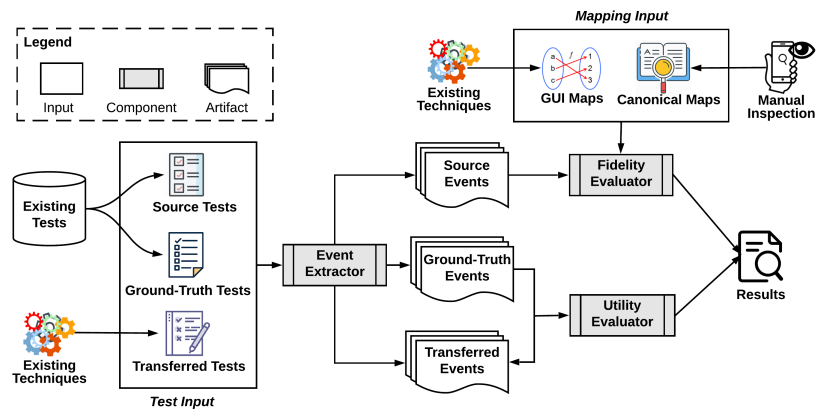

Figure 2: Overview of FrUITeR's automated workflow.

Refining utility's definition with extended metrics is worthy of further study, but outside our scope. Our goal was to show that utility is important and measurable, to motivate further exploration of such important aspect that has been missed by prior work.

\subsection{FrUITeR's Workflow}

To address $\mathrm{Req}_{2}$, Req 3 , and partially Req from Section 3, we designed an automated evaluation workflow with customizable components, shown in Figure 2. The goal of FrUITeR's workflow is to generate reproducible evaluation results for a test-reuse technique's core functionality. The workflow's automation is enabled by two key aspects: (1) the uniform representation of the inputs and artifacts needed in the evaluation process, and (2) a set of customizable components that output the evaluation results of interest automatically.

4.2.1 Uniform Representation of Inputs. As Figure 2 shows, FrUITeR takes two types of input: Test Input (bottom-left) and Mapping Input (top-right). The two are a combination of inputs taken and artifacts produced by existing test-reuse techniques, as well as three new inputs introduced in FrUITeR to automate the evaluation process: Ground-Truth Tests, GUI Maps and Canonical Maps.

Test Input contains source tests, ground-truth tests, and transferred tests as defined in Section 2.1. The tests may be captured in various forms by a test-reuse technique, and we cannot assume that they will be analyzable in a standard way a priori. For instance, all tests in ATM [21] and GTM [20] are represented as Espresso tests [6] in Java, while CraftDroid [25]'s source tests are written in Python using Appium [2] and its transferred tests are represented in JSON [15]. In order to enable their automated evaluation, the heterogeneous tests that are part of FrUITeR's Test Input thus need to be standardized. FrUITeR's Event Extractor converts the Test Input into a standardized representation of source events, ground-truth events, and transferred events as detailed in Section 4.2.2.

Mapping Input consists of the GUI Map and the Canonical Map, which enable automated evaluation of a test-reuse technique's $f i$ delity. The two maps are newly introduced by FrUITeR and captured using a standardized representation. The GUI Map contains the GUI event mapping from a source app to a target app generated by a given test-reuse technique, and is used to compute the fidelity metrics introduced in Section 4.1.1. Prior work does not provide GUI Maps, but only the final Transferred Tests. The events in these tests cannot be used to calculate fidelity by comparing with source events directly, because the transferred events may include ancillary and null events. We further illustrate how we extract the GUI Maps from 
existing techniques and evaluate their fidelity automatically with FrUITeR in Section 5. On the other hand, the Canonical Map contains the mapping from app-specific events to canonical events. This map is manually constructed and is used as the ground-truth mapping for FrUITeR's Fidelity Evaluator component discussed below. Note that AppFlow [23] can generate a Canonical Map automatically using ML techniques. However, AppFlow's certain mapping results can be wrong, and thus cannot be used as the ground truth.

4.2.2 Customizable Components. FrUITeR introduces three customizable components, shown as shaded boxes in Figure 2: Event Extractor, Fidelity Evaluator, and Utility Evaluator.

Event Extractor leverages program analysis to extract the GUI event sequence from the usage tests' code. The sequence is represented as each event's ID or XPath, depending on which of the two is used in the test. ID and XPath are widely used to locate specific GUI elements in tests in various domains, including Android apps [7] and web apps [14]. For simplicity, we will use "ID" to refer to either the ID or XPath of a specific event in the rest of the paper.

To extract the event sequence, Event Extractor analyzes the Test Input to locate the program point of each event based on its corresponding API, e.g., click [4] or sendKeys [10] for tests written with Appium [2]. Once it identifies the location, Event Extractor determines the event's caller, i.e., the GUI element where the event is triggered, and performs a def-use analysis [19] to trace back the definition of the caller's ID. This definition is specified in a given API of the testing framework, such as findElementByld() in Appium [18]. In that case, the def-use analysis is used to pinpoint the findElementByld() call that corresponds to the event's caller so that ID's value can be determined. The input value associated with the event (if any) is determined by def-use analysis in the same manner. In the end, the converted Source Events, Ground-Truth Events, and Transferred Events are represented in a uniform way with IDs regardless of what testing framework is used.

Note that if the Test Input is written in different programming languages or testing frameworks, multiple Event Extractor instances need to be implemented if not available previously. However, this is a one-time effort, and subsequent work can reuse an existing Event Extractor when applied on the tests written in the same language and testing framework. Furthermore, the Event Extractor is easily customizable to process tests written with different frameworks by replacing the relevant APIs' signatures. For instance, when identifying an event caller's ID, the relevant API is findElementByld() if using Appium [2] to test mobile apps, or findElement() if using Selenium $[16,17]$ to test web apps. By simply replacing the relevant API signature, the Event Extractor will be able to process tests written in both Appium and Selenium frameworks.

Fidelity Evaluator takes the Source Events produced by Event Extractor and Mapping Input, and automatically outputs the sets of (1) correct, (2) incorrect, (3) missed, and (4) nonExist cases for calculating FrUITeR's seven fidelity metrics (recall Table 1).

Algorithm 1 describes Fidelity Evaluator in detail. The algorithm iterates through each source event to determine to which of the four cases it should be assigned (Lines 2-16). To do so, it first gets the current source event $(s r c)$, and the transferred event mapped from it (trans) based on the GUI Map (Lines 3-4). It then converts the appspecific events src and trans into their corresponding canonical

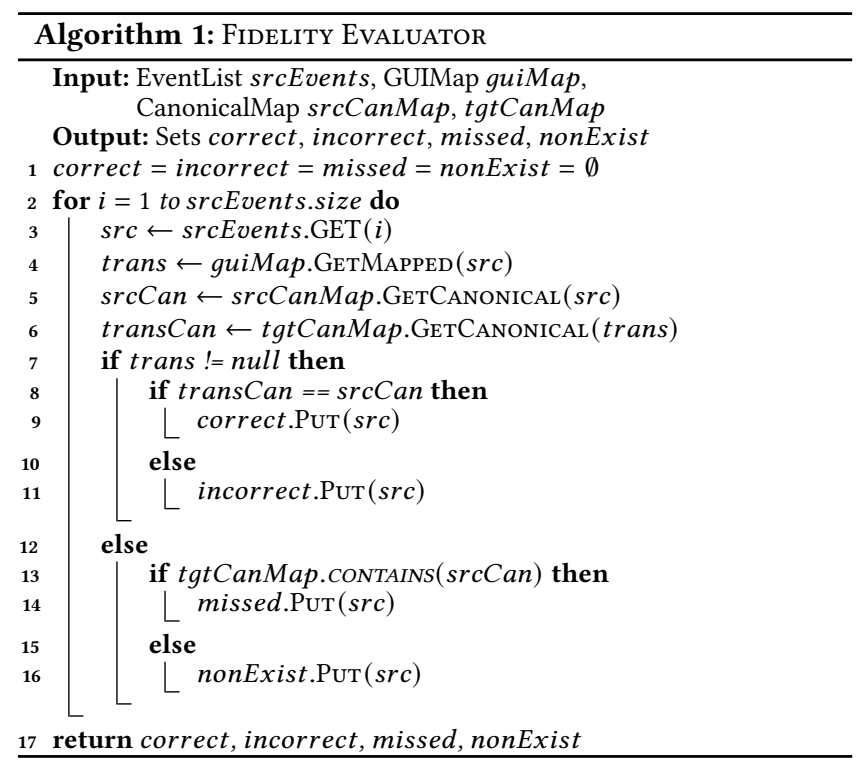

events srcCan and transCan, using their respective Canonical Maps, so that the events are comparable (Lines 5-6). Finally, to determine which of the four cases src falls into, the algorithm first checks whether trans is a null event. If not, transCan will be compared against srcCan to determine whether the transferred event refers to the same canonical event as the source event, and $\operatorname{src}$ will be added to either the correct or incorrect set accordingly (Lines 7-11). If trans is null, the source event has not been mapped to any events in the target app. The algorithm then iterates through the Canonical Map of the target app (tgtCanMap) to determine whether the matching event $\operatorname{srcC}$ an exists in the target app, and $\operatorname{src}$ will be added to either the missed set or nonExist set accordingly (Lines 12-16).

Utility Evaluator automatically analyzes the Ground-Truth Events and Transferred Events produced by Event Extractor. It uses this information to compute the two utility metrics-effort and reduction-based on their definitions described in Section 4.1.2.

4.2.3 Relationship to FrUITeR's Requirements. FrUITeR's workflow yields three key benefits that directly target $R_{e q}, R_{2} q_{3}$, and $\mathrm{Req}_{4}$ introduced in Section 3.

First, the only manual effort required by FrUITeR is to construct the Canonical Maps by relating app-specific events to canonical events. This is a one-time effort per app, and each event only needs to be labeled once regardless of how many times it appears in a test $\left(\mathrm{Req}_{2}\right)$. By contrast, in previous work [20, 21, 25], each app-specific event needs to be manually labeled every time it appears in a test, possibly resulting in thousands of manual inspections.

Second, FrUITeR establishes ground truths with uniform representations: Canonical Maps are the ground truth for assessing fidelity, while Ground-Truth Events help to assess utility. This renders the evaluation results yielded by FrUITeR reproducible ( $\left.\mathrm{Req}_{3}\right)$. For instance, any mistakes or subjective judgments made in the current techniques' manual evaluation processes can be easily located by inspecting the Canonical Maps, and independently reproduced. Further, FrUITeR's Canonical Maps are reusable, modifiable, and extensible for subsequent studies, helping to avoid duplicated work. 


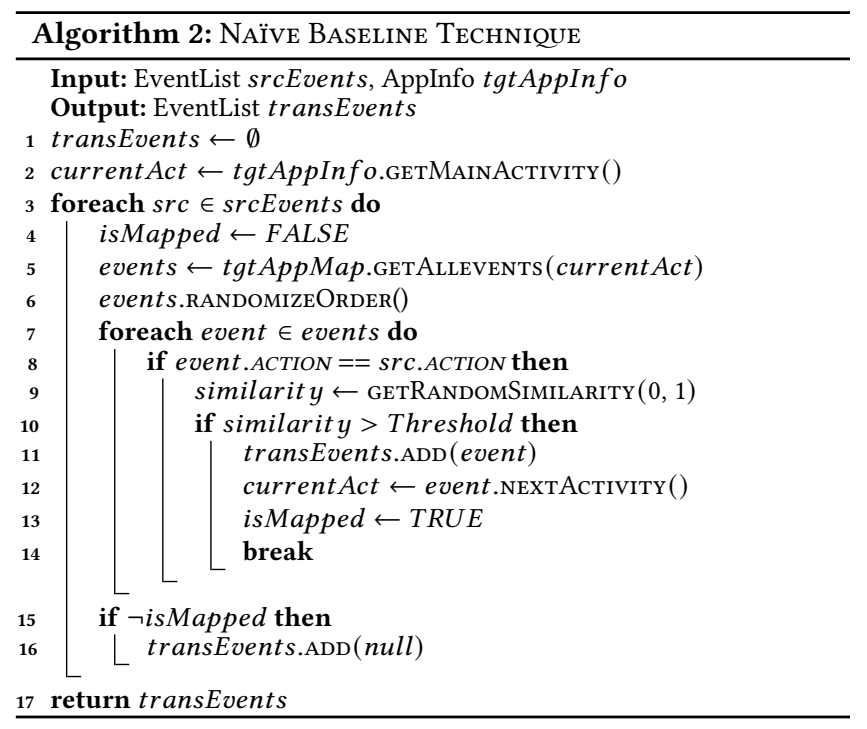

Third, FrUITeR's workflow consists of customizable modules that isolate the evaluation to a relevant component of a test-reuse technique $\left(\mathrm{Req}_{4}\right)$. For instance, Fidelity Evaluator only assesses the performance of GUI event mapping, instead of evaluating a technique as a whole. Moreover, both Fidelity Evaluator and Utility Evaluator can be customized, reused, or extended to automatically evaluate other metrics of interest based on the standardized inputs and artifacts that FrUITeR defines, directly fostering future research.

\subsection{FrUITeR's Baseline Techniques}

To better understand the performance of a test-reuse technique, we developed two baseline techniques-Naïve and Perfect-that establish the lower- and upper- bounds achievable by the fidelity and utility metrics in a given scenario.

4.3.1 Naïve Baseline. The Naïve baseline uses a random strategy to select the events in a target app to which each source event should be mapped. This sets the practical lower-bound of fidelity. As Algorithm 2 shows, Naïve initially explores the target app from the main Activity [9] (Line 2). For each source event, it obtains all the events at the current Activity (events) in a random order (Lines 5-6), and then tries to find a match between the current source event $s r c$ and each event in events (Lines 7-14). When mapping src to event, Naïve first checks if the associated actions of the two events are the same, and only computes the similarity score when they are. The similarity score is computed by selecting a random value between 0 and 1 (Line 9), which are the lower and upper bounds used in existing work. If the similarity score of $\operatorname{src}$ and event is above a certain threshold, event is added to the list maintained in transEvents (Line 11). At that point, Naïve continues to explore the target app from the Activity reached by the transferred event (Line 12), and marks the current source event $s r c$ as mapped (Line 13). In the end, if the source event is not mapped, it will be marked as a null event and added to transEvents (Line 15-16). Null events correspond to either the True Negative or False Negative categories in Table 1.

4.3.2 Perfect Baseline. The Perfect baseline transfers the source events based on the ground-truth mapping we establish (recall
Section 4.2), assuming all source events are correctly mapped to the target app. Perfect baseline thus represents a "perfect" GUI event mapping and always achieves $100 \%$ fidelity by definition. Specifically, we are interested in the utility achieved by the Perfect baseline since it represents the upper-bound of the transferred tests' practical usefulness, which is not considered by existing work. This can help us identify the room for improvement and guide future research in test-reuse techniques. We will discuss our findings regarding this in Section 6.

\section{FrUITeR's INSTANTIATION}

This section describes how we instantiate FrUITeR to automatically evaluate the relevant modules of existing techniques alongside FrUITeR's baseline techniques, in partial satisfaction of Req 4 . The evaluation is performed based on FrUITeR's reusable benchmark that addresses Req 5 . To this end, we needed to provide information that enables FrUITeR's automated workflow discussed in Section 4.2 and depicted in Figure 2: the Source Tests that are supplied as inputs to a given test-reuse technique; the Transferred Tests and GUI Maps, which are produced as outputs of a given test-reuse technique; and the manually constructed ground truths, namely, Canonical Maps and Ground-Truth Tests. However, existing test-reuse techniques were not designed with FrUITeR's modular workflow in mind, and thus do not provide such information directly.

Section 5.1 explains how we mitigated the above challenge in order to extract the relevant components from existing techniques and generate the information needed by FrUITeR. Note that this step will not be necessary for future techniques if they follow FrUITeR's modularized design. Section 5.2 presents FrUITeR's reusable benchmark for the uniform evaluation of test-reuse techniques, which contains the Source Tests, Ground-Truth Tests, and Canonical Maps used in FrUITeR's automated workflow. Finally, Section 5.3 provides the details of FrUITeR's implementation and generated datasets.

\subsection{Modularizing Existing Techniques}

To lay the foundation for addressing Req $_{4}$, we modularized FrUITeR's design. In turn, this isolated the evaluation of GUI event mapping's fidelity and the transferred tests' utility, as discussed in Section 4.2. However, the existing techniques are implemented and evaluated as fully integrated, one-off solutions that do not provide the artifacts needed by FrUITeR to generate the modularized evaluation results. Because of this, we had to extract the specific functionality from existing techniques' implementations that performs the GUI event mapping (recall Section 2.2). Once the GUI Maps are available, we can generate the Transferred Tests used in FrUITeR's Utility Evaluator. Note that the step of extracting GUI Mapper components is not needed for future test-reuse techniques if they follow FrUITeR's modularized design. For example, we directly applied FrUITeR on the two baseline techniques we developed, with no extra effort.

Extracting the GUI Mapper components from the existing techniques was challenging since we had to understand each technique's design and implementation in detail, and to modify its source code. To this end, in addition to the available publications, we studied in depth the existing approaches' implementations $[1,3,5,8]$ and communicated with their authors extensively. We describe the challenges we faced during this process and the specific componentextraction strategies we applied to each existing solution. 
5.1.1 Extracting AppFlow's GUI Mapper. AppFlow [23] is an ML-based technique whose key component trains a classifier that maps app-specific events to canonical events, but does not map the events from a source app to a target app. To compare AppFlow with similarity-based techniques, we leverage its Canonical Maps to transfer the source events to the target app by (1) mapping each source event to the corresponding canonical event based on the source app's Canonical Map and (2) mapping this canonical event back to the app-specific event in the target app based on the target app's Canonical Map. AppFlow's implementation does not output its Canonical Maps, so we had to locate and modify the relevant component to do so. Moreover, AppFlow does not store its trained classifier, so we had to configure its ML model and re-train it. During this process, we communicated with AppFlow's authors closely to understand its code, to obtain proper configuration files and training data, and to ensure the correctness of our re-implementation.

5.1.2 Extracting ATM's and GTM's GUI Mappers. As discussed earlier, ATM [21] was developed as an enhancement to GTM [20] and was shown to outperform it [21]. However, the authors of these two techniques compared them only on half of the source-target app pairs used in ATM's publication [21] due to the large manual effort required. Since FrUITeR largely automates the comparison process, we decided to extract the GUI Mapper components from both techniques to enable their comparison at a large scale.

An obstacle we had to overcome was that ATM and GTM both require the app's source code due to their use of the Espresso framework [6]. Thus, they cannot be compared as-is with techniques evaluated on closed-sourced apps, which would have limited our choice of benchmark apps. We discussed this issue with ATM's and GTM's authors and learned that the only step that requires source code for both techniques' GUI Mappers is computing the textual similarity score of image GUI elements (e.g., ImageButton). In that case, the text of the image's filename is retrieved from the app's code and analyzed to compute the similarity score. However, the main author confirmed that, in her experience, this feature is rarely needed in practice. We thus decided to extract ATM's and GTM's GUI Mapper components as stand-alone Java programs that do not require Espresso, omitting the filename-retrieval feature. We subsequently confirmed with the two techniques' authors the correctness of our implementation.

5.1.3 Extracting CraftDroid's GUI Mapper. CraftDroid's [25] implementation is only partially available. Its authors informed us that two of CraftDroid's modules-Test Augmentation and Model Extraction-were not releasable when we requested them, due to ongoing modifications, while the prior versions of the two modules were no longer available. The authors confirmed our observation that CraftDroid's GUI mapping functionality depends on the outputs of the two missing modules, and advised us that the best strategy would be for us to reimplement them based on CraftDroid's lone publication [25]. However, the publication in question is missing a number of details that would introduce bias in our re-implementation: we would have no guarantee that the versions of the two components we produce are the same as those used in CraftDroid. Instead, we decided to rely on CraftDroid's published Transferred Tests [5] in our evaluation.
To obtain CraftDroid's GUI Maps, we inspected its published artifacts [5] and found that only certain events in the Transferred Tests have associated similarity scores, while other events are labeled as "empty". Further investigation showed that each event in the Transferred Tests belongs to one of three cases: (1) events with available similarity scores are successfully mapped from the source events; (2) "empty" events are mapped from the source events but no match is found by CraftDroid (i.e., null events); (3) the remaining events are not mapped from the source events but are added by CraftDroid (i.e., ancillary events). We excluded the ancillary events so that the resulting transferred events have a 1-to-1 mapping from the source events, giving us CraftDroid's GUI Maps.

\subsection{FrUITeR's Benchmark}

As discussed above in the motivation for Req ${ }_{5}$, existing test-reuse techniques are evaluated on different apps and tests, which hinders their comparability. To address this, we established a reusable benchmark with the same apps and tests to serve as a shared measuring stick in this emerging domain. This section discusses our strategy for including existing apps and tests in the benchmark, and for generating the required ground truth.

5.2.1 Benchmark Apps and Tests. To maximize the results from existing work that we can attempt to reproduce, we first included the intersection of the subject apps used by existing work. This yielded 3 shopping apps: Geek, Wish, and Etsy. We further randomly selected 7 additional shopping apps and 10 news apps used by AppFlow [23]. This gave us 20 benchmark apps in total, as described in Table 2. Our rationale behind this choice of apps was two-fold: (1) AppFlow's authors manually inspected all app categories on Google Play and identified shopping and news as categories with common functionalities suitable for test reuse; (2) AppFlow was evaluated on the largest number of subject apps among the existing techniques. By comparison, ATM [21] used 16 open-source apps that are not as popular as those used in AppFlow.

To construct the benchmark tests, we further followed the test cases defined in AppFlow, with a similar rationale: (1) AppFlow's authors conducted an extensive study to manually identify tests that are shared in shopping and news apps; (2) AppFlow defines a larger number of tests compared to other work. For example,

Table 2: Summary information of benchmark apps.

\begin{tabular}{|c|c|c|c|c|c|}
\hline \multirow{7}{*}{} & App ID & App Name & \#Downloads & \#Tests & \#Events \\
\cline { 2 - 6 } & S1 & AliExpress & $100 \mathrm{M}$ & 15 & 76 \\
\cline { 2 - 6 } & S2 & Ebay & $100 \mathrm{M}$ & 13 & 48 \\
\cline { 2 - 6 } & S3 & Etsy & $10 \mathrm{M}$ & 13 & 55 \\
\cline { 2 - 6 } & S4 & 5miles & $5 \mathrm{M}$ & 12 & 78 \\
\cline { 2 - 6 } & S5 & Geek & $10 \mathrm{M}$ & 13 & 85 \\
\cline { 2 - 6 } & S6 & Google Shopping & $1 \mathrm{M}$ & 15 & 72 \\
\cline { 2 - 6 } & S7 & Groupon & $50 \mathrm{M}$ & 14 & 66 \\
\cline { 2 - 6 } & S8 & Home & $10 \mathrm{M}$ & 14 & 98 \\
\cline { 2 - 6 } & S9 & 6PM & $500 \mathrm{~K}$ & 14 & 63 \\
\cline { 2 - 6 } & S10 & Wish & $100 \mathrm{M}$ & 14 & 85 \\
\hline \multirow{5}{*}{ News } & N1 & The Guardian & $5 \mathrm{M}$ & 13 & 76 \\
\cline { 2 - 6 } & N2 & ABC News & $5 \mathrm{M}$ & 9 & 31 \\
\cline { 2 - 6 } & N3 & USA Today & $5 \mathrm{M}$ & 11 & 28 \\
\cline { 2 - 6 } & N4 & News Republic & $50 \mathrm{M}$ & 10 & 40 \\
\cline { 2 - 6 } & N5 & BuzzFeed & $5 \mathrm{M}$ & 11 & 50 \\
\cline { 2 - 6 } & N6 & Fox News & $10 \mathrm{M}$ & 11 & 28 \\
\cline { 2 - 6 } & N7 & SmartNews & $10 \mathrm{M}$ & 9 & 20 \\
\cline { 2 - 6 } & N8 & BBC News & $10 \mathrm{M}$ & 9 & 22 \\
\cline { 2 - 6 } & N9 & Reuters & $1 \mathrm{M}$ & 10 & 37 \\
\cline { 2 - 6 } & N10 & CNN & $10 \mathrm{M}$ & 9 & 24 \\
\hline
\end{tabular}


CraftDroid [25] only has 2 tests defined in each app category. We excluded those tests that require mocking external dependencies (e.g., a payment service). This resulted in 15 tests in the shopping category and 14 tests in the news category, shown in Table 3. Note that we cannot reuse AppFlow's tests directly because they are written in a special-purpose language defined by AppFlow for an entire app category rather than a specific app. Instead, we relied on multiple undergraduate and graduate students with Android experience to write the applicable tests for each of the 20 subject apps using Appium [2]. Some benchmark apps did not have each functionality described in Table 3, ultimately resulting in a total of 239 tests involving 1,082 events across the 20 apps (the two right-most columns of Table 2), requiring 3,920 SLOC of Java code.

These benchmark tests currently do not contain oracle events because only ATM [21] and CraftDroid [25] can transfer oracles in principle. However, due to the limited availability of CraftDroid's source code as mentioned earlier, we would not be able to obtain CraftDroid's results using our benchmark, making a comparison across different techniques impossible. As additional test-reuse techniques are developed with the ability to transfer oracles, FrUITeR's benchmark tests can be extended to include oracle events to obtain their results as well. Note that as long as future techniques follow FrUITeR's modularized design to provide the needed input (e.g., GUI Maps of the oracle event mapping), FrUITeR will be able to automatically generate the results of oracle events. A detailed tutorial is provided on FrUITeR's website [12].

5.2.2 Benchmark Ground Truth. As described in Section 4.2, we define Canonical Maps to represent the ground truth for the fidelity of the GUI event mapping, and Ground-Truth Events to represent the ground truth for the utility of the transferred tests.

In our benchmark, we define 72 canonical events for the shopping apps and 55 for the news apps. Our canonical events are extended from AppFlow, aiming to reflect a finer-grained classification of GUI events. For instance, event "password" in the sign-in test (TS1/TN1 in Table 3), and events "password" and "confirm password" in the sign-up test (TS2/TN2 in Table 3), are all represented as the same canonical event "Password" in AppFlow. However, it is debatable whether that is appropriate. For example, mapping "password" in

Table 3: Benchmark test cases in shopping (TS) and news (TN) categories.

\begin{tabular}{|c|c|c|}
\hline Test ID & Test Case Name & Tested Functionalities \\
\hline TS1/TN1 & Sign In & provide username and password to sign in \\
\hline TS2/TN2 & Sign Up & provide required information to sign up \\
\hline TS3/TN3 & Search & use search bar to search a product/news \\
\hline TS4/TN4 & Detail & find and open details of the first search result item \\
\hline TS5/TN5 & Category & find first category and open browsing page for it \\
\hline TS6/TN6 & About & find and open about information of the app \\
\hline TS7/TN7 & Account & find and open account management page \\
\hline TS8/TN8 & Help & find and open help page of the app \\
\hline TS9/TN9 & Menu & find and open primary app menu \\
\hline TS10/TN10 & Contact & find and open contact page of the app \\
\hline TS11/TN11 & Terms & find and open legal information of the app \\
\hline TS12 & Add Cart & add the first search result item to cart \\
\hline TS13 & Remove Cart & open cart and remove the first item from cart \\
\hline TS14 & Address & add a new address to the account \\
\hline TS15 & Filter & filter/sort search results \\
\hline TN12 & Add Bookmark & add first search result item to the bookmark \\
\hline TN13 & Remove Bookmark & open the bookmark and remove first item from it \\
\hline TN14 & Textsize & change text size \\
\hline
\end{tabular}

sign-up to "password" in sign-in may lead to non-executable tests. To remove ambiguity, we capture such events separately.

Based on the canonical events, we construct 20 Canonical Maps, one per subject app. We do so by manually relating to the canonical events a total of 561 subject apps' GUI events that appear in one or more of the 239 tests. As discussed in Section 4.2, this is the only manual step required by FrUITeR and is a one-time effort: the Canonical Maps can be reused when relying on the same subject apps. As a point of comparison, recall from Section 3 that evaluating 48 app pairs in ATM [21] required manually inspecting 2,400 events. By contrast, our one-time inspection of the 561 events enabled the use of 200 app pairs ( 2 categories $\times 10 \times 10$ apps, i.e., including an app's test transfer to itself) by every technique FrUITeR evaluated.

The Ground-Truth Events in our benchmark are extracted from the 239 tests by FrUITeR's Event Extractor (recall Figure 2).

\subsection{FrUITeR's Implementation Artifacts}

FrUITeR's artifacts are publicly available [12]: its source code; final datasets; GUI Mappers extracted from existing work; implementations of baseline techniques, their GUI Maps, and Transferred Tests; benchmark apps and tests; and manually constructed benchmark ground truths. We highlight the key details of these artifacts below.

5.3.1 Source Code. FrUITeR's Event Extractor (recall Figure 2) is implemented in Java using Soot [11] (235 SLOC). FrUITeR's Fidelity Evaluator and Utility Evaluator are implemented in Python (1,045 SLOC). FrUITeR's baseline techniques Naïve and Perfect (recall Section 4.3) are likewise implemented in Python (112 SLOC). The GUI Mapper components extracted from existing techniques (recall Section 5.1) are implemented in their original programing languages: AppFlow in Python (1,084 SLOC); GTM in Java (1,409 SLOC); and ATM in Java (1,314 SLOC). The functionality that processes their outputs and generates the uniform representation of GUI Maps and Transferred Tests is implemented in Python (404 SLOC). As discussed earlier, due to CraftDroid's unavailable source code, we can only interpret its published artifacts [5]; that functionality is implemented in Python (86 SLOC). The data analyses that interpret our final datasets are written in R (585 SLOC).

5.3.2 Final Datasets. Our final datasets contain the results of 11,917 test transfer cases generated by the GUI Mappers from the existing techniques and our two baselines when applied on FrUITeR's benchmark. We apply 5 techniques-AppFlow, ATM, GTM, Naïve, Perfect-to transfer tests across 20 shopping and news apps, involving 1,000 source-target app pairs ( 2 app categories $\times 100$ app pairs in each category $\times 5$ techniques). This yielded 2,381 result entries per technique. As discussed earlier, we have to rely on CraftDroid's final results, and can thus only compare CraftDroid to the other techniques on the 3 shopping apps-Geek, Wish, Etsy-used both in our benchmark and in CraftDroid's evaluation. This gave us 12 result entries for CraftDroid since only 2 tests are transferred by CraftDroid in each app. Each of the total 11,917 result entries contains the following information: (1) the source and target apps; (2) the source, transferred, and ground-truth tests; (3) the technique used to transfer the test; (4) the correct/incorrect/missed/nonExist sets of GUI events output by FrUITeR's Fidelity Evaluator as described in Algorithm 1, and the seven corresponding fidelity metrics 


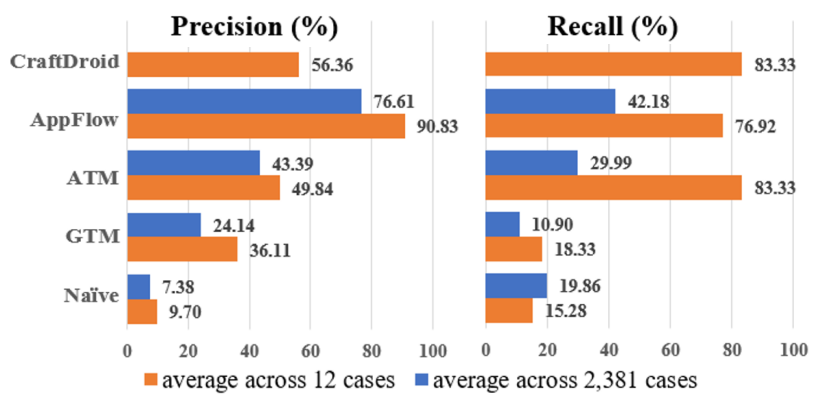

Figure 3: Comparison of average precision and recall.

defined in Section 4.1.1; and (5) values of the two utility metricseffort and reduction-defined in Section 4.1.2. Note that obtaining these 11,917 result entries following prior work's evaluation processes would have required manual inspection of 53,963 events that appear across all of the source tests, which is infeasible in practice.

\section{FINDINGS}

The datasets produced by FrUITeR include the results obtained by evaluating side-by-side the extracted GUI Mapper components from the four existing test-reuse techniques [20,21, 23, 25] and the two baseline techniques we developed. In turn, this data enables further in-depth studies of a range of research questions in this emerging domain. As an illustration, this section highlights several findings uncovered by FrUITeR's datasets that are missed by prior work.

\subsection{GUI Mapper Comparison}

As discussed earlier, existing techniques are evaluated in their entirety, on different benchmark apps and tests, and using different evaluation metrics, all of which makes their results hard to compare. By contrast, FrUITeR was able to evaluate their extracted GUI Mappers side-by-side, with our two techniques-Naïve and Perfectserving as baselines. We note that it is possible for a given test-reuse technique to produce results as a whole that may be different from those produced only by its extracted GUI Mapper. One reason may be that there is additional relevant functionality that is scattered across the technique's implementation. However, any such functionality can be added to the existing GUI Mappers, or introduced in additional FrUITeR components.

6.1.1 Fidelity Comparison. FrUITeR's website [12] contains the results of all seven fidelity metrics from Section 4.1.1 obtained using our benchmark. Due to space limitations, we show the results of three fidelity metrics (Precision, Recall, Accuracy), and restrict our discussion to Precision and Recall since Accuracy follows a similar trend as Precision; the results of the four remaining fidelity metrics (Correct, Incorrect, Missed, NonExist) can provide an indepth understanding on each of the four specific cases, and can be found on FrUITeR's website [12]. Figure 3 shows the average precision and recall achieved by the four existing techniques as well as Naïve; we omit Perfect since its values are always $100 \%$ by definition.

For each technique except CraftDroid, the top (blue) bar shows the average calculated based on 2,381 cases transferred among both shopping and news apps. To meaningfully compare CraftDroid with

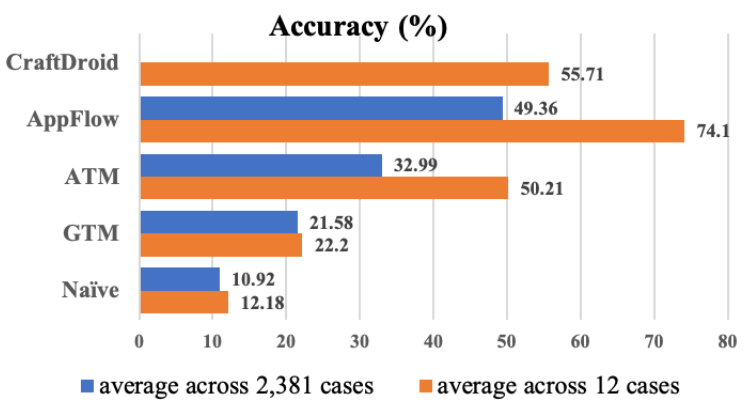

Figure 4: Comparison of average accuracy.

other techniques, even if only partially, we show the averages calculated based on the 12 cases for which we have CraftDroid's data, in the bottom (orange) bars. CraftDroid only transferred "Sign In" and "Sign Up" tests in the 3 shopping apps-Geek, Wish, and Etsyleading to the 12 cases ( 6 source-target app pairs $\times 2$ tests).

We highlight three observations based on the results from Figure 3. First, every existing technique yields lower recall than precision on the larger (blue) data set, meaning that it suffers from more missed (i.e., false negative) than incorrect (i.e., false positive) cases. Although AppFlow's recall is highest among the existing techniques, it exhibits the largest drop-off between its precision and recall values. A plausible explanation is that, as an ML-based technique, AppFlow will likely fail to recognize relevant GUI events if no similar events exist in its training data. This was somewhat unexpected, however, given that AppFlow's authors carefully crafted its ML model to the app categories we also used in FrUITeR, and suggests that additional research is needed in selecting and training effective ML models for UI test reuse. By comparison, similaritybased techniques such as ATM will miss fewer GUI events in principle: they can always compute a similarity score between two events and return the mapped events whose scores are above a given threshold. However, if the similarity threshold is set too low, it will result in more incorrect cases, leading to low precision.

A related observation is that AppFlow's precision outperforms the other techniques across the board, for both the larger (blue) and smaller (orange) datasets. This is because AppFlow has the advantage of more information, obtained from a large corpus of apps in its training dataset, than the similarity-based techniques, which compute the similarity scores based only on the information extracted from the source and target apps under analysis. However, AppFlow's recall is lower than both ATM and CraftDroid on the 12 (orange) cases from Geek, Wish, and Etsy. This reinforces the above observation that an ML-based technique will fail to recognize GUI events if no similar events exist in its training data.

Finally, our data confirms that ATM indeed improves upon GTM, as indicated in their pairwise comparisons across both precision and recall, on both large and small datasets. In fact, GTM exhibits the lowest fidelity of all existing techniques, and its recall across the 2,381 (blue) cases is actually lower than that achieved by the Naive strategy. We note that GTM's design is geared to transferring tests in programming assignments that share identical requirements, and is clearly not suited to heterogenous real-world apps.

6.1.2 Utility Comparison. Figure 5 shows the two utility metrics yielded by each of the four existing and two baselines. Recall from 


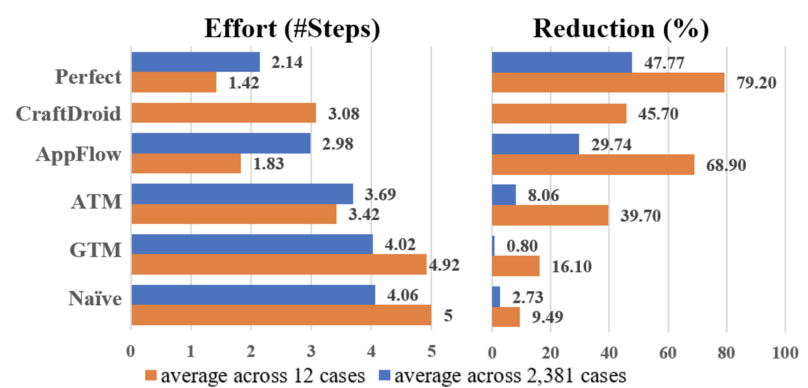

Figure 5: Comparison of average effort and reduction.

Section 4.1.2 that utility measures how useful the transferred tests are in practice compared to the ground-truth tests. The objective of utility is to minimize the effort while maximizing the reduction.

The utility of existing techniques shows similar trends to those observed in the case of fidelity. For example, AppFlow outperforms other techniques, while GTM exhibits similar performance to that of Naïve. This indicates a possible correlation between the fidelity of the GUI event mapping and the utility of the transferred tests.

At the same time, we observe that, while our Perfect GUI Mapper achieves higher utility than the remaining techniques, that utility is not optimal. In fact, Perfect's average reduction is under $50 \%$ across the 2,381 cases in the larger dataset (top, blue bar). In other words, even with the best possible mapping strategy, we save less than half of the effort required to complete the task manually. The previously published techniques perform much worse than this: AppFlow saves under $30 \%$, ATM under $10 \%$, and GTM under $1 \%$ of the required manual effort, while the reduction yielded by CraftDroid on the smaller (orange) dataset is lower than Perfect's on either of the two datasets. This indicates that fidelity is clearly not the only factor to consider in order to achieve desired utility, and that there is large room for improvement in future test-reuse techniques.

To verify the above insights, we conducted pairwise correlation tests between the seven fidelity and two utility metrics. Overall, the results, further discussed below and provided in their entirety in FrUITeR's online repository [12], show a weak correlation between fidelity and utility. This reinforces our observation that accurate GUI mappings can yield useful transferred tests, but are not the only relevant factor. In turn, this finding calls for exploration of other components in test-reuse techniques since the focus on GUI event mapping alone can hit a "ceiling", as shown by the Perfect baseline. We discuss such possible directions next.

\subsection{Insights and Future Directions}

Guided by the above observations, we explore potential strategies for improving UI test reuse with various statistical tests and manual inspections on FrUITeR's datasets. Due to space limitations, we highlight four findings that were not reported by previous work.

Source app selection matters for a given target app. Figures 3, 4, and 5 all show consistent improvement across the techniques in the smaller datasets (12 cases transferred among 3 apps) compared to the larger ones (2,381 cases transferred among 20 apps). This suggests that certain source-target app pairs achieve better results than others. For example, we found that app pairs involving Wish, Geek, and a benchmark app called Home-all of which are developed by the same company, Wish Inc--achieve high fidelity and utility, regardless of the technique used. Another such compatible app pair is ABC News and Reuters. Performing a large-scale evaluation enabled by FrUITeR will help spot pairings like this, and give researchers a starting point to explore the characteristics that can lead to better transfer results.

Automated transfer is not suitable for all tests. Our utility metrics revealed large effort and negative reduction in some cases, meaning that correcting a transferred test required more work than writing it from scratch. Further inspection revealed that this is primarily due to a test's length rather than a technique's accuracy. For instance, Perfect showed no benefit (reduction $\leq 0$ ) $16 \%$ of the time, and the average number of source events in those cases is only 4 . This suggests that, for simple tests, manual construction may be preferable. Future research should consider the criteria for suitable tests to transfer instead of transferring all source tests.

There is a trade-off between ML- and similarity-based techniques. As discussed above, an insufficient training set in an MLbased technique may yield low recall, while a low similarity threshold in a similarity-based technique can address this but may yield low precision. This suggests two future research directions. First, selecting training sets and similarity thresholds is important, but existing techniques did not justify their choices [20, 21, 23, 25]. There is clearly a need for further study of novel strategies such as incorporating dynamic selection criteria based on target app characteristics. Second, future research should consider the tradeoffs across different test-reuse techniques and provide guidance on selecting the most suitable techniques for a given scenario.

Test length is not a key factor influencing fidelity. CraftDroid and GTM studied the relationship between the test length and their transferred results. For instance, CraftDroid showed a strong negative correlation between test length and its two fidelity metrics (coefficient $<-0.5$ in both cases). To verify these findings, we conducted correlation tests on FrUITeR's much larger datasets. Our results indicate a negative but very weak correlation between test length and FrUITeR's fidelity metrics $(-0.25<$ coefficient $<0$ across all seven cases). This shows that test length is not the key factor that impacts fidelity, arguing that future research targeting reuse of complex tests may be a fruitful direction.

\section{CONCLUSION}

This paper has presented FrUITeR, a customizable framework for automatically evaluating UI test-reuse techniques. FrUITeR has been instantiated and successfully demonstrated on the key functionality extracted from existing test-reuse techniques that target Android apps. In the process, we have been able to identify several avenues of future research that prior work has either missed or actually flagged as not viable. We publicly release FrUITeR, its accompanying artifacts, and all of our evaluation data, as a way of fostering future research in this area of growing interest and importance.

\section{ACKNOWLEDGMENTS}

This work is supported by the U.S. National Science Foundation under grants 1717963 and 1823354, U.S. Office of Naval Research under grant N00014-17-1-2896, and ERC Advanced Fellowship Grant no. 741278 (EPIC). 


\section{REFERENCES}

[1] 2019. AppFlow's source code and artifacts. https://github.com/columbia/appflow.

[2] 2019. Appium: Mobile App Automation Made Awesome. http://appium.io

[3] 2019. ATM's source code and artifacts. https://sites.google.com/view/ apptestmigrator/.

[4] 2019. Click - Appium. http://appium.io/docs/en/commands/element/actions/ click

[5] 2019. CraftDroid's source code and artifacts. https://sites.google.com/view/ craftdroid/.

[6] 2019. Espresso. https://developer.android.com/training/testing/espresso.

[7] 2019. Find Elements - Appium. http://appium.io/docs/en/commands/element/ find-elements

[8] 2019. GTM's source code and artifacts. https://sites.google.com/view/ testmigration/.

[9] 2019. Introduction to Activities | Android Developers. https://developer.android. com/guide/components/activities/intro-activities

[10] 2019. Send Keys - Appium. http://appium.io/docs/en/commands/element/ actions/send-keys

[11] 2019. Soot - A Java optimization framework. https://github.com/Sable/soot.

[12] 2020. FrUITeR's website. https://felicitia.github.io/FrUITeR/.

[13] 2020. Gherkin Syntax - Cucumber Documentation. https://cucumber.io/docs/ gherkin

[14] 2020. How to locate an element on the page - Web Performance https://www.webperformance.com/load-testing-tools/blog/articles/realbrowser-manual/building-a-testcase/how-locate-element-the-page

[15] 2020. JSON - Wikipedia. https://en.wikipedia.org/wiki/JSON

[16] 2020. SeleniumHQ Browser Automation. https://www.selenium.dev

[17] 2020. Web element :: Documentation for Selenium. https://selenium.dev/ documentation/en/webdriver/web_element
[18] admin. 2019. Chapter-4: Appium Locator Finding Strategies - Kobiton. Kobiton (Apr 2019). https://kobiton.com/book/chapter-4-appium-locator-findingstrategies

[19] Frances E. Allen and John Cocke. 1976. A program data flow analysis procedure. Commun. ACM 19, 3 (1976), 137.

[20] Farnaz Behrang and Alessandro Orso. 2018. Test migration for efficient largescale assessment of mobile app coding assignments. In Proceedings of the 27th ACM SIGSOFT International Symposium on Software Testing and Analysis.

[21] Farnaz Behrang and Alessandro Orso. 2019. Test Migration Between Mobile Apps with Similar Functionality. In 34th International Conference on Automated Software Engineering (ASE 2019).

[22] Shauvik Roy Choudhary, Alessandra Gorla, and Alessandro Orso. 2015. Automated test input generation for android: Are we there yet? (E). In 2015 30th IEEE/ACM International Conference on Automated Software Engineering (ASE).

[23] Gang Hu, Linjie Zhu, and Junfeng Yang. 2018. AppFlow: using machine learning to synthesize robust, reusable UI tests. In Proceedings of the 2018 26th ACM foint Meeting on European Software Engineering Conference and Symposium on the Foundations of Software Engineering. ACM, 269-282.

[24] Vladimir I Levenshtein. 1966. Binary codes capable of correcting deletions, insertions, and reversals. In Soviet physics doklady, Vol. 10. 707-710.

[25] Jun-Wei Lin, Reyhaneh Jabbarvand, and Sam Malek. 2019. Test Transfer Across Mobile Apps Through Semantic Mapping. In 34th International Conference on Automated Software Engineering (ASE 2019).

[26] Mario Linares-Vásquez, Carlos Bernal-Cárdenas, Kevin Moran, and Denys Poshyvanyk. 2017. How do developers test android applications?. In 2017 IEEE International Conference on Software Maintenance and Evolution (ICSME).

[27] Christopher D Manning, Prabhakar Raghavan, and Hinrich Schütze. 2008. Introduction to information retrieval. Cambridge university press.

[28] Andreas Rau, Jenny Hotzkow, and Andreas Zeller. 2018. Transferring Tests Across Web Applications. In Web Engineering, Tommi Mikkonen, Ralf Klamma, and Juan Hernández (Eds.). Springer International Publishing, Cham, 50-64. 